\title{
Keyword index to volume 34
}

Activities of daily living 729

Acute transverse myelitis 481

Acute transverse myelopathy 633

Adhesive arachnoiditis 403

$\mathrm{ADL}$, see Activities of daily living

Aetiology 549

American Spinal Injury Association motor score 531

Angiogenesis 394

Angiography, coronary 308

Anterior decompression and fusion 387

Apophyseal ring 427

Arachnoiditis, adhesive 403

Arm cranking 684

Arm ergometry 167

Ascorbic acid 592

Ashworth scale 560

ASIA, see American Spinal Injury Association motor score

Assessment 560

Atelectasis 54

Atlanta paralympics 433

Auditory vigilance 573

Australia 639

Automatic dysreflexia 173

Axonal regeneration 320, 394

Bacteriuria 587

Balanitis 243

Behçet's disease 305

Bladder biopsy 137

Bladder inhibition 95

Bladder neck dyssynergia 34

Bone mineral density 736

Book reviews 181-182, 566-568

Brain injury 351

Broadband ultrasound attenuation 736

Bulbocavernosus reflex 95

C3/4 spinal cord injury 486

C-fibres 137

Calipers, long leg 666

Catecholamines 264

Catheterization

clean intermittent 284

sterile urethral 30

suprapubic 93

Cauda equina 116

Cauda equina syndrome 427

Central cord syndrome 176

Central pain 707

Central pattern generator 91

Cerebrospinal fluid $\mathbf{4 2 2}$

Cervical disc protrusion 488

Cervical myelopathy 220

Cervical spinal cord injury 326

Cervical spinal stenosis 620

Cervical spine 358, 536, 620, 699

Cervical spondylosis 711
Children 176

Chordoma 358

Chronic spinal cord lesions 65

CIC, see Clean intermittent catheterization

Circulation 422

Clean intermittent catheterization 284

Clinical correlation 65

Cognition 107

Colonic transit time 277

Complications 152, 470

Compressive myelopathy 100

Continuous positive airway pressure 54

Control 525

Control measures 315

Conus medullaris 116, 630

Coronary angiography 308

Corrigenda 123

Cortisol 264

Costs 470

Cryptic vascular malformation 268

Cystoscopy 651, 691

Cysts

hydatid 297

sacral hyatid 361

Decompression surgery 220,387

Decubitus ulcer 255

Deformity of spine, following surgery 536

Destination after discharge 679

Detrusor hyperreflexia 95

Diagnosis 100

Diaphragm 167, 673

Diaphragm fatigue 594

Diaphragm function 626

Dietary fibre 277

Disability 531

Disaster 382

Dog motoneurons 320

Dual energy X-ray absorptiometry 736

Dynamometer, isokinetic 46

Dysplasia, vesical 578

Dyssynergia, bladder neck 34

Dystrophy, reflex sympathetic 239

Earthquake 382

Echinococcal infection 361

Editorials 381

EGFRs, see Epidermal growth factor receptors

Elderly patients 220

Electrical stimulation 8, 95, 411

Electro-ejaculation 696

Electromyography 158, 594

Endoscopic submeatal paste 657

Endoscopic surgery 361

Endoscopic treatment 34

Energy expenditure 659
Epidemiology 152, 416, 549, 608, 679

Epidermal growth factor receptors 578

Epinephrine 264

Ergometry, arm 167

Event-related potentials 107

Exercise 127

Expansive cervical laminoplasty 725

Experimental models 449

External fixation 113

False urethral passage 651

Feneley procedure 93

FES, see Functional electrical stimulation

Fiji 549

Flexible cystoscope 651

Folic acid 460

Follow-up 752

Fracture dislocations 1

Functional electrical stimulation 8, 24, $127,264,507,611,615,659$

Functional independence measure 531

Functional neuromuscular stimulation 16

Fusion 387

Gait 8, 659

Gluteus maximus flap 346

Gymnastic trauma 427

Hand function 326

Handstand 176

Handwashing 30

Head position 602

Health services 639

Hematomyelia 268

Hindbrain hernia 720

Hydatid cysts 297

Hydrocephalus 699

Hydroxyapatite 725

Hyperreflexia, detrusor 95

Hyperplasia 137

Immunohistochemistry 137

Immunosuppression 82

IMSOP, see under International Medical Society of Paraplegia

Incidence 549

Incomplete paraplegia 308

Incomplete spinal cord injury 620

Incremental field test 288

Integrated electromyogram 158

Intermittent catheterisation 742

International Medical Society of

Paraplegia, AGM 249-250

Intervertebral disc protrusion 716

Intervertebral foramen 644

Intravesical instillation 284

Ischaemia, spinal cord 308

Isokinetic dynamometer 46 
Kobe-Osaka, Japan 382

Laminoplasty 699

expansive surgical 725

Laser sphincterotomy 227

Laser surgery 227

Léger and Boucher test 288

Length of stay 679

Letters to the Editor $60,121,180$, 498-500, 754-755

Liaison psychiatry 752

Lipid peroxidation 234

Lipoma, thoracic 633

Locomotion 507

Long leg calipers 666

Lower extremities 239

Lumbar radiculopathy 644

Lumbar spinal canal stenosis 403

Lumbar spine 1, 427

Lumbosacral spine 644

MAG3, see Tc-99m-mercaptoacetyltriglycerine

Magnetic resonance imaging 65, 193, 214, $220,268,630,720$

Magnetic transcranial stimulation 673

Manual muscle test 543

Manubrium splitting approach 387

Measurement 560

Mechanical ventilation 626

Medical bladder augmentation 284

Meetings and Notices 62-63, 124-126, 183-185, 251-253, 311-313, 366-379, 437-448, 569-571, 635-637, 703-705, 757-759

Memorial Lecture, in honour of Dr Ailie Key 466-469

Message from the President 187

Metaphasia 578

Methicillin-resistent Staphylococcus aureus 315

Microsurgery 644

Micturition disturbance 481

Mortality 752

Motor cortex 673

Motor evoked potentials 272, 673

Motor recovery 188, 711

Motor scores 531

Motorneurons, dog 320

MRI, see Magnetic resonance imaging

MRSA, see Methicillin-resistent Staphylococcus aureus

Multiple sclerosis 351

Muscle action potential 39

Muscle force 24

Muscle tone 46

Myelitis, acute transverse 481

Myelodysplasia 493

Myelomeningocele 460

Myelopathy 488

acute transverse 633

cervical 220

compressive 100

thoracic 387

Myeloscopy 403

Myocutaneous flap 346
Myometer 543

Neoplasms

spinal 536, 633

vesical 578

Nerve growth factor 394

Neural tube defects 460

Neurography 326

Neurological examinations 193

Neurological prognosis 100, 620

Neurological recovery 164

Neurological standards 193

Neuromuscular stimulation, functional 16

Neuropathic bladder 137, 227, 284, 305, 578, 587, 657

Neurophysiology 193

Neurosarcoidosis 116

NGF, see Nerve growth factor

NK cell function 82

Non-traumatic paraplegia 549

Norepinephrine 264

Obituaries 59, 179, 365, 501-502, 565

Omental transposition 193

OPLL, see Ossification of the posterior longitudinal ligament

Orthoses 507

reciprocating gait 611,659

Orthotic hip joint 611

Ossification 699

of the posterior longitudinal ligament 387

Osteocartilaginous endplate 427

Osteomyelitis, panvertebral 113

Outcomes 152, 470

Outcome measures 16

Oxybutynin hydrochloride 284

Oxygen consumption 684

Oxygen uptake 167

P300 107

Pain syndromes 239

Panvertebral osteomyelitis 113

Paralympics, Atlanta 433

Paralysis 255

Paraparesis 116

Paraplegia 8, 24, 82, 91, 107, 113, 127 , $288,338,460,507,611,615,666,684$

Pathology of spinal cord injuries 1

PDWHF, see Platelet derived wound healing formula

Peak oxygen uptake 288

Pediatric spinal cord injury 669

Pediatrics 16

Pelvic floor musculature 411

Pelvis fracture 382

Penile erection 691

Perceived exertion 684

Perineal contraction 95

Perineal electrical stimulation 411

Periodic limb movements 294

Peripheral nerve grafting 320

Phenoxybenzamine 239

Phrenic nerve 626

Physical activity 294

Physical strain 729
Physical work capacity 684, 729

Plasmid analysis 587

Platelet derived wound healing formula 394

Poliomyelitis 204, 748

Poliovirus vaccine 748

Posterior fossa decompression 720

Posterior longitudinal ligament 699

Posture 158

Pott's paraplegia 272

Pregnancy 696

Pressure sores and ulcers 255, 346

Prostatectomy 227

Psychiatric morbidity 669

Psychological defences 669

Psychological profiles 351

Pulmonary function tests 602

Pulsation, spinal cord 422

Pyuria 742

Radiculopathy 427, 488, 644

Radiofrequency 214

Rat 234

Reciprocating gait orthoses 611,659

Referees for Paraplegia 1994/1995 247

Reflex sympathetic dystrophy 239

Rehabilitation 351, 525, 531, 666, 748

Rehabilitation medicine 679

Renal clearance 338

Renal studies 338

Repair 449

Reproducibility 338

Researches 449

Respiratory muscles 204, 594, 673

Respiratory weaning 626

Restless legs syndrome 294

Restriction fragment length polymorphism 587

Retrograde axonal tracing 320

Rib fracture 382

Risk factors 255

Rupture 651

Sacral hyatid cysts 361

Salbutamol 691

Scalenes 673

Secondary conditions 470

Sensory deprivation 573

Sleep 294

Somatosensory and motor evoked potentials 272

Somatosensory perception 107

Spasticity 560

Speed of sound 736

Sphincterotetomy, laser 227

Spina bifida 460

Spinal canal stenosis 486

Spinal circuitry 91

Spinal cord 268, 416, 422, 630

Spinal cord compression 333

Spinal cord disease 24

Spinal-cord-evoked potentials 100

Spinal cord injuries $16,24,30,34,39,46$, $65,93,95,107,113,127,137,152$, $158,164,173,188,193,214,234$, $243,255,277,294,301,315,320$, 
$351,394,411,433,449,470,507$, $525,531,543,549,560,573,578$, $587,592,608,639,651,666,669$, $691,696,736,742,752$

Spinal cord injury complications 255

Spinal cord injury with radiographic abnormality 176

Spinal cord ischaemia 308

Spinal cord lesions 91, 536, 659, 679 chronic 65

Spinal cord pulsation 422

Spinal cord stenosis 333

Spinal cord trauma 301

Spinal cysts 536

Spinal disease 297, 707

Spinal fracture 382

Spinal infection 716

Spinal injury 707

Spinal manipulation 488

Spinal neoplasms 536, 633

Spinal paralysis 297

Spinous process 725

Spondylosis

cervical 711

thoracic 333

Sports activity 416, 433, 729

Standing 127, 615, 666

Standing frame 666

Stenosis 403, 620, 644

cervical spinal 620

spinal canal 486

spinal cord 333

Sterile urethral catheterisation 30

Stool weight 277
Stress 82

Stress fracture 173

Suicide 752

Suprapubic catheterisation 93

Surgery 346

Surgical decompression 100, 644

Surgical treatments 188,620

Surveillance 639

Surveys 639

Syringohydromyelia 301

Syringomyelia 301, 536, 720

T-cells 243

T-cell function 82

Tc-99m-mercaptoacetyltriglycerine 338

TES, see Therapeutic electrical stimulation

Testicular sperm 696

Testosterone 264

Tetraplegia 54, 82, 127, 167, 173, 204, $239,326,338,602,626,691,711,729$

Thailand 608

Therapeutic electrical stimulation 24

Thermal regulation 433

Thermal stress 433

Thoracic lipoma 633

Thoracic myelopathy 387

Thoracic spine 1, 333

Thoracic spondylosis 333

Tracheotomy 486

Transcranial magnetic stimulation 39

Transurethral resection 34

Trauma 416

Treatment 297

Trunk support 167
Tuberculoma 630

Tuberculosis of the spine $272,630,716$

Ultrasonography 422,736

Unstable spinal fracture 486

Upper thoracic spine 387

Urethra 651, 742

Urethral closure 93

Urethral opening pressure 493

Urethral strictures 227

Urinary acidifying agent 592

Urinary bladder 227

Urinary incontinence 411

Urinary tract deterioration 493

Urinary tract infection 30, 592, 742

Urodynamic assessment 305

Urodynamic study 481

Vaccination campaign 748

Ventilation 602

Ventilatory failure 204

Vertebral fracture 164

Vesical dysplasia and metaphasia 578

Vesical neoplasia 578

Vesical outlet obstruction 34

Vesical urothelial proliferation 578

Vesicoureteric reflux 657

Vitamin C 234

Vitamin E 234

Walking 615, 666

Wheelchair exercise 288

Wheelchair sports 433

Wheelchair users 351 